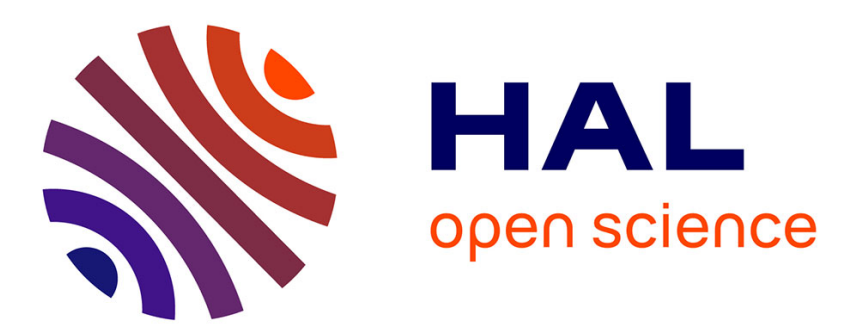

\title{
An Analytical Model for Performance Analysis of an Active Signaling-based TDMA MAC Protocol for Vehicular Networks
}

Fouzi Boukhalfa, Mohamed Hadded, Paul Mühlethaler, Oyunchimeg Shagdar

\section{- To cite this version:}

Fouzi Boukhalfa, Mohamed Hadded, Paul Mühlethaler, Oyunchimeg Shagdar. An Analytical Model for Performance Analysis of an Active Signaling-based TDMA MAC Protocol for Vehicular Networks. VTC-FALL 2019 - IEEE Vehicular Technology Conference, Sep 2019, Honolulu, Hawaii, United States. hal-02178355v2

\author{
HAL Id: hal-02178355 \\ https://hal.science/hal-02178355v2
}

Submitted on 10 Jul 2019

HAL is a multi-disciplinary open access archive for the deposit and dissemination of scientific research documents, whether they are published or not. The documents may come from teaching and research institutions in France or abroad, or from public or private research centers.
L'archive ouverte pluridisciplinaire HAL, est destinée au dépôt et à la diffusion de documents scientifiques de niveau recherche, publiés ou non, émanant des établissements d'enseignement et de recherche français ou étrangers, des laboratoires publics ou privés. 


\title{
An Analytical Model for Performance Analysis of an Active Signaling-based TDMA MAC Protocol for Vehicular Networks
}

\author{
Fouzi Boukhalfa*, Mohamed Hadded*, Paul Muhlethaler ${ }^{\dagger}$ and Oyunchimeg Shagdar* \\ ${ }^{*}$ Institute VEDECOM, 23 bis allée des Marronniers, 78000 Versailles, France \\ ${ }^{\dagger}$ EVA Team, Inria Paris, 2 Rue Simone Iff, 75012 Paris, France \\ \{fouzi.boukhalfa, mohamed.elhadad, oyunchimeg.shagdar\}@vedecom.fr, paul.muhlethaler@inria.fr
}

\begin{abstract}
In Vehicular Ad hoc NETworks (VANETs) the vehicles moving along roads communicate with each other through ad hoc wireless devices. VANETs have attracted a great deal of attention in the research community in recent years, with the main focus being on their support of safety applications. Time Division Multiple Access (TDMA)-based protocols are advantageous in many aspects of VANETs. They can cope with the hidden-terminal problem, and guarantee a strict Quality-ofService $(\mathrm{QoS})$ to satisfy real-time applications. However, the initial assignment of time-slots to the vehicles can suffer from the access collision problem, which can frequently occur between vehicles trying to access the same time slots. Moreover, a low latency access is not usually possible. That is why we have developed an Active Signaling system (AS-DTMAC : Active Signaling Decentralized Tdma MAC protocol) which operates above the existing DTMAC protocol : a Medium Access Control (MAC) protocol specially devoted to VANETs. AS-DTMAC can drastically reduce the number of access collisions and also offer low latency access. The aim of this article is to provide a complete mathematical analysis of the performance of this scheme, to show its high performances and to validate these results using simulations.
\end{abstract}

Keywords-VANETs, TDMA, Active signaling, Low latency, 5G, Analytical model, Network simulation.

\section{INTRODUCTION AND MOTIVATION}

Vehicular Adhoc NETworks (VANETs) have received considerable attention both in academic communities and industrial companies [1]. They aim to establish communication and collaboration between vehicles, and a number of safety, commercial and entertainment applications [2] have been developed. With the coming of autonomous driving, future networks will need to enable real-time services with immediate reaction to enhance safety. These applications must satisfy specific and stringent QoS and safety requirements. To meet these requirements, efficient Medium Access Control (MAC) protocols must be developed [2] in order to handle network access and transmission with minimum packet loss. One of the best known techniques used in the proposed solutions with a deterministic protocol (to guarantee a bounded delay) is Time Division Multiple Access (TDMA), which allows a number of vehicles to share the same frequency channel by dividing the channel into different time slots. Each vehicle can access the channel during its dedicated time slot to send data messages, whereas it can only receive messages during the time slots reserved for other vehicles. Our proposed protocol called AS-DTMAC [4] operates on a fully distributed TDMA for VANETs, strengthened by active signaling as a mechanism to prevent access collisions. To the authors' best knowledge using active signaling in a TDMA scheme is one of the first protocols to combine the advantages of random access (i.e. small access delay for sporadic traffic) and the advantage of TDMA (stability of the access rights and hidden collision avoidance). The aim of this paper is to propose an analytical model of this new scheme in order to estimate the residual collision rate of this protocol and other performance metrics.

The paper is organized as follows. Section II presents related work. Section III recalls the DTMAC protocol [3] and the enhanced version AS-DTMAC with their main features. In Section IV, we introduce the analytical model and simulation results for three different performance metrics. Finally, Section $\mathrm{V}$ concludes this paper and discusses future work.

\section{RELATED WORK}

The channel access techniques are of prime importance for the performance of VANETs. For Medium Access Control (MAC) protocols, the literature has distinguished two kinds of protocols: contention-based and contention-free. In contention-based protocols, the vehicles randomly access the channel when they need to transmit. This kind of protocol allows multiple access to the channel based on channel sensing [5]. But, for critical-applications, the lack of reliability of the broadcast mechanism means that the transmission of safety messages with bounded access delay is very challenging. For instance, this is true for the emerging standard in VANETs: IEEE802.11p [6], which uses a random algorithm based on both Enhanced Distributed Channel Access (EDCA) and Carrier Sense Multiple Access with Collision Avoidance (CSMA/CA) mechanisms [7]. For real-time applications, it is therefore more suitable to use the second category i.e. contention-free access. With these schemes, in a given neighborhood, there is only one vehicle which can access the channel at any given time. These protocols are deterministic and provide a bounded access delay with collision-free transmission. In recent years, a considerable number of such distributed MAC protocols have been proposed in order to guarantee low latency access and reliable communication for safety applications in VANETs. However, these schemes can suffer from access collision problem which occurs when two or more vehicles within the same two-hop neighborhood set attempt to access the same available time slot, a problem which is likely to happen when a distributed scheme is used [2] and merging collision problem which occurs when 
two vehicles in different two-hop sets accessing the same time slot become members of the same two-hop set due to changes in their position. Consequently all the contributions tend to focus on these problems which occur particularly in mobility scenario. For example, the hidden-exposed terminal problem has been overcome by the authors of the Medium Access Control protocol ADHOC MAC [8]. ADHOC MAC is a distributed contention-free scheme based on R-ALOHA (Reliable R-ALOHA [9]). Each vehicle can access the channel at least once in each frame by randomly selecting a time slot as its Basic CHannel (BCH).

Another well-known algorithm called VeMAC [10] proposes a contention-free multi-channel MAC protocol for VANETs. To solve the problem of hidden nodes, VeMAC grants the slots to the vehicles depending on the direction in which the vehicle is heading (Left;Right). Since VeMAC, like other distributed schemes, suffers from the access collision problem, another protocol called ATSA [11] was proposed which uses VeMAC as background protocol and introduces a more complex mechanism to schedule the slots. It makes the frame length dynamical using a binary tree algorithm to decrease the probability of collisions.

We believe that the AS-TDMAC protocol we introduced in [4] is the first protocol for VANETs which deeply intertwines an advanced random access scheme and a TDMA scheme for VANETs. AS-TDMAC shows the advantages of TDMA in the sense that when a slot is acquired by a vehicle this slot can be re-used synchronously and the transmissions do not suffer from the hidden-node problem as in IEEE 802.11p. In the same time, AS-TDMAC can offer a low latency access to urgent packets, property usually only devoted to random access techniques. The goal of the present paper is to provide a detailed and accurate analytical model of this protocol and to compare the results of this model with simulation results. As a by-product of our analysis, we will show that AS-TDMAC is an excellent access scheme in VANETs with very good performance metrics.

\section{THE DTMAC PROTOCOL WITH ACTIVE SIGNALING}

In this section, we start by a brief description of the DTMAC protocol. In the second subsection, we explain how active signaling operates and how it can be combined with DTMAC. More information on DTMAC and AS-DTMAC can be respectively found in [3] and [4].

\section{A. The DTMAC protocol}

DTMAC is a fully distributed and location-based TDMA. The main feature of this protocol is that it assumes that each vehicle is equiped with a GPS (Global Positioning System) or a GALILEO receiver that allows it to have information of its position (latitude, longitude and altitude) and accurate speed and exact time. The road is split into different zones of length $R$, denoted by $x_{i}, i=1, \ldots, N$, where $R$ is the communication range of the vehicles, and we introduce a spatial slot reuse as shown in Figure 1. We can see that the vehicles in zone $x_{1}$ can use the same set of slots as the vehicles in $x_{4}$. The only rule to this spatial reuse is that the distance between simultaneously transmitting vehicles must be greater than $2 \times R$. Another particularity of this protocol is that each packet sent by the vehicles, contains a special information: the Frame Information in which the vehicle sends the status of the previous slots of the frame (available, busy, in collision). Such information can be used by the vehicles that receive the packet to randomly select a suitable slot in the next frame. Thus DTMAC can be seen as a slotted Aloha protocol on the 'non' busy slots of the frame. The principle of slot allocation in DTMAC is shown in Figure 2. This protocol can support vehicles moving in opposite directions and under varying traffic conditions (speed, density).

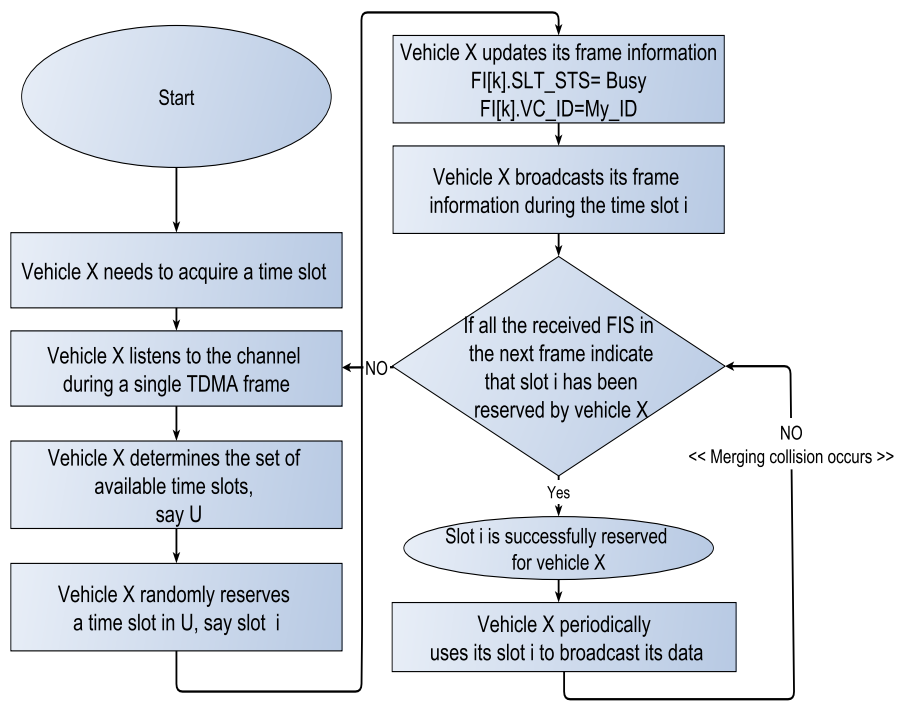

Fig. 2. DTMAC protocol principle

\section{B. Active signaling with DTMAC}

We use DTMAC as the background protocol but to enhance the scheme we add another selection mechanism based on an active signaling technique. Figure 3 illustrates a slot which contains a burst of signaling and the rest of the slot is used to send the payload packet. During the signaling part of the packet, a selection process is carried out to obtain exactly one packet to be sent in the payload part of the slot.

Signaling bursts

Payload part of the packet

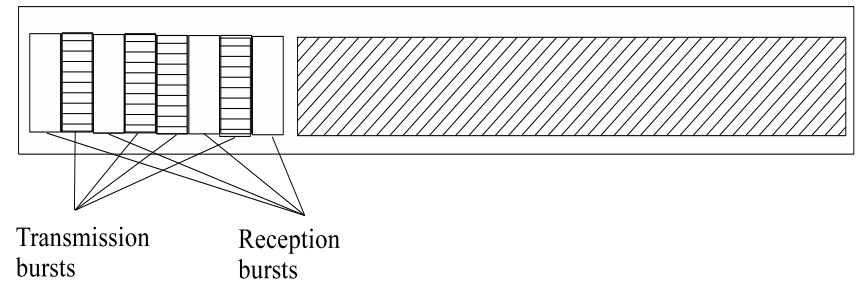

Fig. 3. Slot structure of the Active Signaling mechanism

The burst of signaling consists in a succession of transmission periods and listening periods. During a listening period, when a transmission is sensed by a vehicle that is attempting to send a packet, its transmission attempt is aborted. The pattern of the transmission in the signaling interval follows a binary key. ' 1 ' means that the vehicle with a packet to 


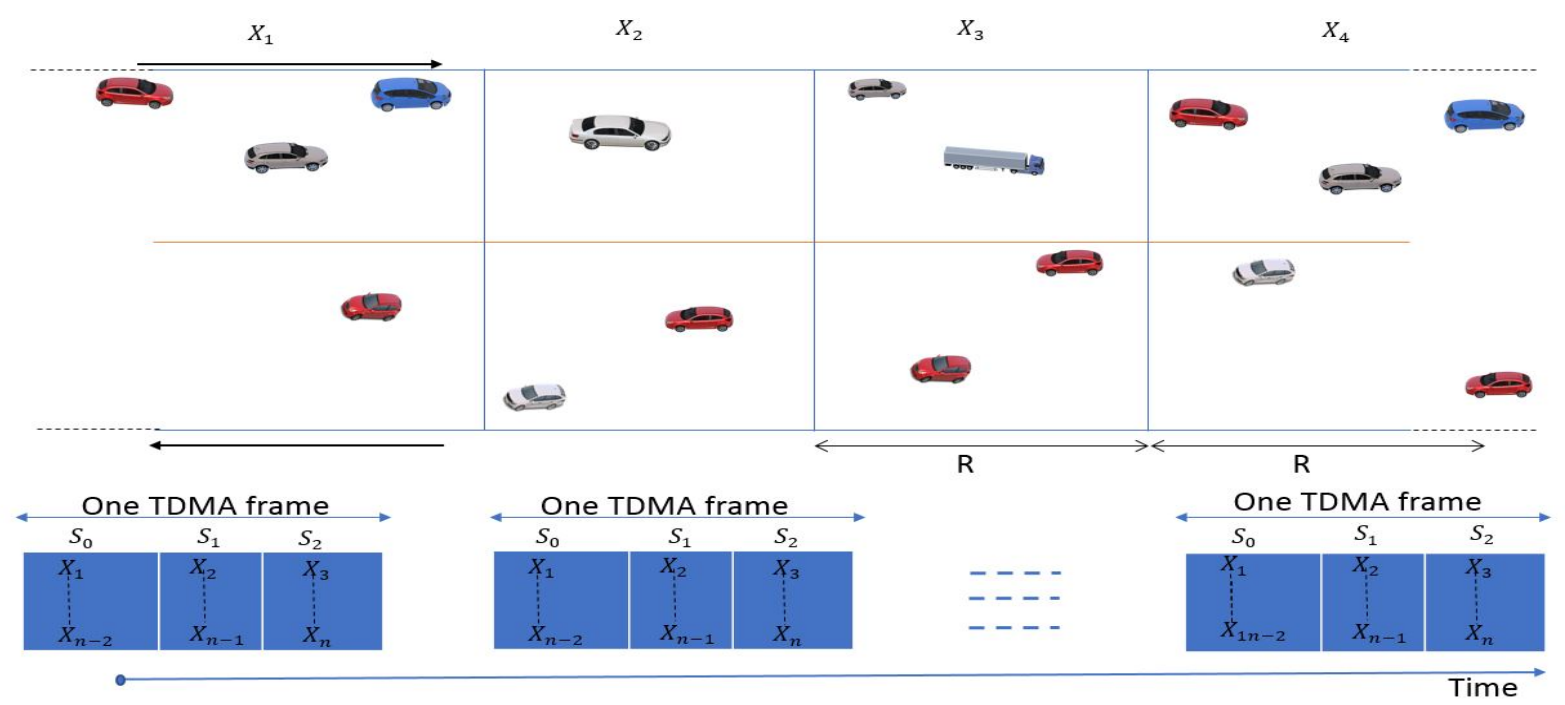

Fig. 1. TDMA slots scheduling principle.

send transmits during the signaling burst. ' 0 ' means that the vehicle with a packet to send senses the channel during this mini-slot of selection. For instance, a vehicle which draws the key '01001110' will listen during the first mini-slot and if no competing transmission is sensed during this mini-slot, it will transmit during the next mini-slot. The two following steps in the selection process will be two listening periods. The selection process continues using the same rule until the key is completely used up, see Figure 4. We have to note that the selection process depicted in this Figure applies in a slot chosen using the random slot selection process of DTMAC (see Figure 2).

In the selection process we have $n$ selection mini-slots. In most cases we will assume that the selection key which encompasses $n$ bits is selected at random. However these keys could be handled using other techniques. Let us suppose that we have 'standard' vehicles trying to send their packets and reserve a slot using the scheme previously described. The first bit of their selection key will always be set to ' 0 '. In contrast, a few 'emergency' vehicles wish to have an immediate access and thus the first bit of their selection key will be set to ' 1 '. With this choice, the 'emergency' vehicles will be be granted access in priority over the 'standard' vehicles.

\section{ANALYTICAL MODEL AND SIMULATION RESULTS}

In this section we build an analytical model for ASDTMAC and compare the performance given by this model to simulation results. For the simulations the scenario is essentially the same as the scenario used in [4]. We will briefly summarize this scenario and the simulation tools we use.

We use MOVE [12] to generate vehicular traffic scenarios and SUMO [13] to perform real vehicular mobility. We consider a real highway area on a digital map to generate a VANET environment close to real highway configurations, taking into account different lane directions. In Figure 5 we can see a metropolitan area taken from a Map of San Jose (California) of size $3000 \mathrm{~m} \times 100 \mathrm{~m}$. This map was exported from OpenStreetMap (OSM) and adapted with the help of

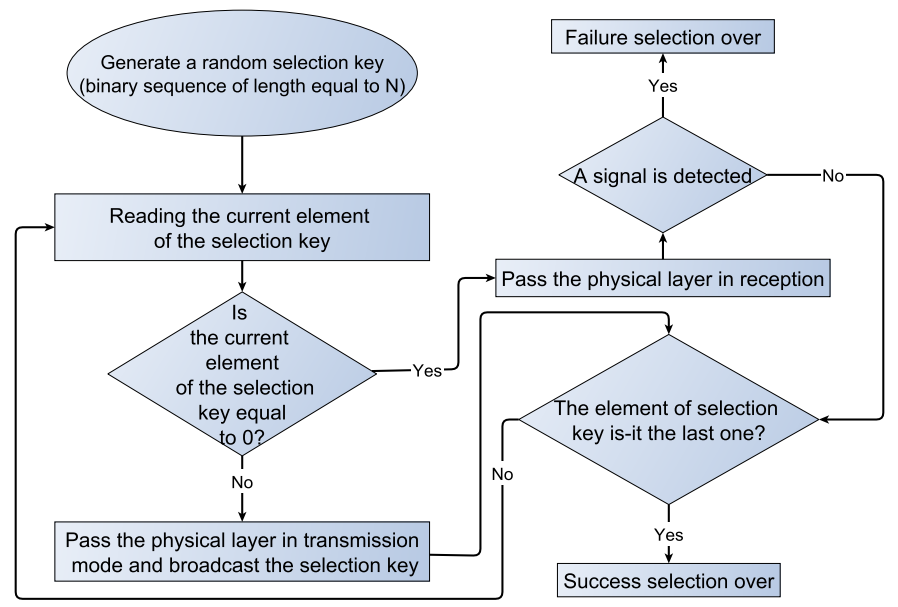

Fig. 4. Active signaling

OpenStreetMap Editor (JOSM). The resulting roads are then populated with vehicles traveling in both directions. Each flow of vehicles is characterized by a set of parameters which consist of the starting and ending time of the flow, the initial point and the destination of the flow and the maximum number of vehicles. In this environment, each vehicle is assigned a random speed between $120 \mathrm{~km} / \mathrm{h}$ and $150 \mathrm{~km} / \mathrm{h}$. The resulting traffic traces generated by MOVE were injected into the Network Simulator $n s 2.34$. Table I summarizes the simulation parameters used in our scenarios.

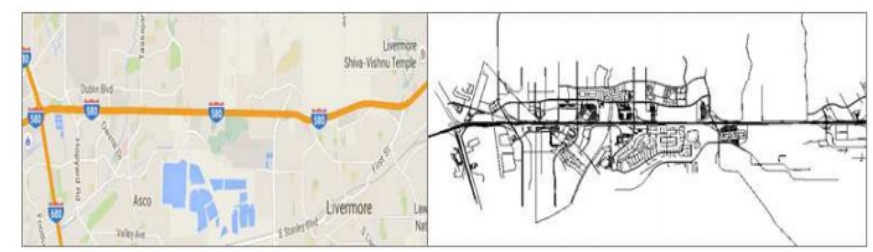

Fig. 5. VANET network topology captured from Google MAP. 
TABLE I. Simulation parameters

\begin{tabular}{cc}
\hline Parameter & Value \\
\hline Simulation duration & $100(\mathrm{~ms})$ \\
Speed & $120(\mathrm{~km} / \mathrm{h})$ \\
Speed standard deviation & $30(\mathrm{~km} / \mathrm{h})$ \\
Number of slots per frame $(\tau)$ & 100 \\
Slot duration & $0.001(\mathrm{~s})$ \\
Highway length & $2.5(\mathrm{~km})$ \\
The number of lanes per direction & 2 \\
The radio range $(R)$ & $310(\mathrm{~m})$ \\
\hline
\end{tabular}

For the model we distinguish two cases: in the first case we have an homogeneous arrival on each slot; in the second case there is a burst of very urgent messages arriving. The first case covers the default case where the vehicles try to randomly select a free slot for their transmissions; active signaling is used to avoid a collision when two or more vehicles select the same slot. This is the access for 'standard' vehicles. The second case is to enable a low latency access to a few sporadic packets. In this second case a special choice of the transmission key must be made in order to favor the urgent packets. This is the access for 'emergency' vehicles. In the last part of this study, we evaluate the speed of convergence of the protocol i.e. the percentage of successfully reserved slots after each of the protocol frames.

\section{A. Homogeneous arrival}

The model uses a random access technique for each free slot. The vehicles use the FI field to discover the available free slots and select one of them at random. Thus the arrival of a transmission on each free slot can be modeled as a Poisson process of rate $\lambda$. The probability that there are $k$ transmission attempts during this slot is:

$$
\frac{\lambda^{k}}{k !} \exp (-\lambda)
$$

We assume that we have a binary key of $n$ bits or, in other words, the signaling scheme encompasses $n$ mini-slot intervals. Our goal is to compute the probability that at the end of the selection only one vehicle has been selected to transmit.

To ease the computation we will assume that the transmission key of a node is randomly generated between 0 and $2^{n}-1$, which means that after each transmission in a mini-slot a vehicle which is still in the selection process will transmit in the next mini-slot with probability $1 / 2$ and will listen with probability $1 / 2$.

Let us suppose that we have $k$ vehicles at the beginning. Our aim is to compute the probability that $j$ vehicles are still in the selection process after the mini-slot $i$, we denote this probability by $a_{i}^{j}$. To perform this task, we use the generating function $A_{i}(x)$ of the remaining contenders after the ith selection mini-slot. By definition we have:

$$
A_{i}(x)=\sum_{j=0}^{\infty} a_{i}^{j} x^{j}
$$

It is easy to establish that

$$
A_{0}(x)=x^{k}
$$

And we can observe that

$$
A_{1}(x)=A_{0}\left(\frac{x}{2}+\frac{1}{2}\right)+A_{0}\left(\frac{x}{2}\right)-A_{0}\left(\frac{1}{2}\right) .
$$

The correction term $A_{0}\left(\frac{x}{2}\right)-A_{0}\left(\frac{1}{2}\right)$ is just to take into account the fact that when the $k$ vehicles select a listening period, none of them is rejected by the selection process and we still have $k$ contenders after this step of the selection process.

Similarily we have the recursion for $i \in 0, \ldots, n-1$ :

$$
A_{i+1}(x)=A_{i}\left(\frac{x}{2}+\frac{1}{2}\right)+A_{i}\left(\frac{x}{2}\right)-A_{i}\left(\frac{1}{2}\right)
$$

Thus using this recursion formula, it is easy to compute $A_{1}(x), A_{2}(x), \ldots, A_{n}(x)$ for instance using Maple. To show one example, we can fix $k=3$ and $n=5$. We obtain the results shown in Table II below. After the 5th round of mini-slot selection, the probability that there are still three contenders is $\frac{1}{1024}$, the probability that there are still two contenders is $\frac{93}{2048}$ and exactly one contender $\frac{1953}{2048}$. If the selection process stops after the 5 th round the probability that the process is successful is :

$$
\frac{1953}{2048} \simeq 0.954
$$

and the probability of collision is:

$$
1-\frac{1953}{2048} \simeq 0.0464
$$

\begin{tabular}{|c|c|c|c|c|}
\hline i $a_{k}^{i}$ & $a_{0}^{i}$ & $a_{1}^{i}$ & $a_{2}^{i}$ & $a_{3}^{i}$ \\
\hline$i=0$ & 0 & 0 & 0 & 1 \\
\hline$i=1$ & 0 & $\frac{3}{8}$ & $\frac{3}{8}$ & $\frac{1}{4}$ \\
\hline$i=2$ & 0 & $\frac{21}{32}$ & $\frac{9}{32}$ & $\frac{1}{16}$ \\
\hline$i=3$ & 0 & $\frac{105}{128}$ & $\frac{21}{128}$ & $\frac{1}{64}$ \\
\hline$i=4$ & 0 & $\frac{465}{512}$ & $\frac{45}{512}$ & $\frac{1}{256}$ \\
\hline$i=5$ & 0 & $\frac{1953}{2048}$ & $\frac{93}{2048}$ & $\frac{1}{1024}$ \\
\hline
\end{tabular}

TABLE II. DIFFERENT PHASES OF THE SELECTION PROCESS

In the general case, the probability of collision after the end of the signaling period is

$$
\sum_{j=2}^{\infty} a_{j}^{n}=1-a_{0}^{n}-a_{1}^{n}=1-A_{n}(0)-A_{n}^{\prime}(0)
$$

since $\sum_{j=0}^{\infty} a_{j}^{n}=1$. We can notice that $A_{n}(0)=a_{0}^{n}=0$. Thus if we denote by $A_{n}^{k}(x)$ the generating functions obtained with the above recursive procedure starting with $A_{0}(x)=x^{k}$, we 
can obtain $P_{n}(\lambda)$ the probability of collision with a signaling burst of length $n$ given that we have at least one vehicle attempting to transmit during a slot. We have the following formula:

$P_{n}(\lambda)=\frac{1}{1-\exp (-\lambda)} \sum_{k=0}^{\infty} \frac{\lambda^{k}}{k !} \exp (-\lambda)\left(1-A_{n}^{k}(0)-A_{n}^{k^{\prime}}(0)\right)$.

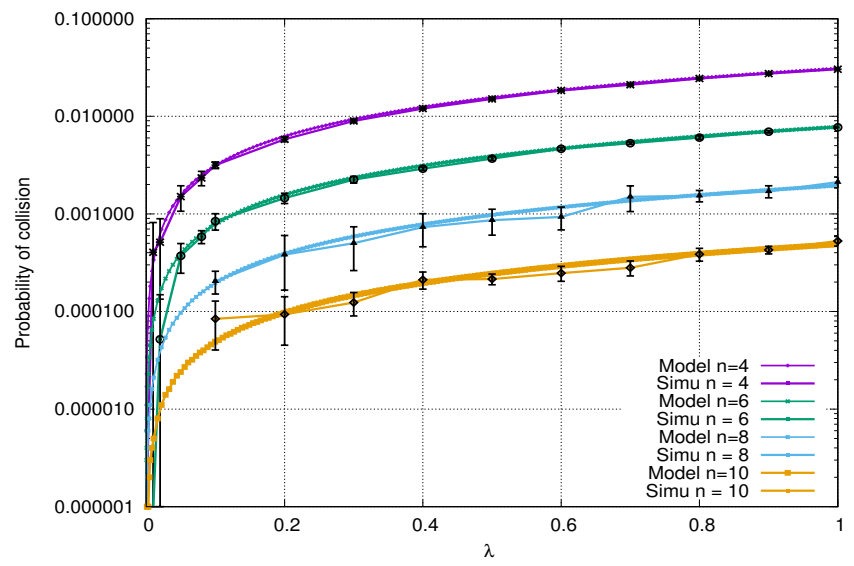

Fig. 6. Collision probability versus channel load.

We use the model developed above to compute the collision probability versus the channel load. In Figure 6, we have the collision probability versus the channel load for $n=4,6,8,10$. The error bars in black are for a $95 \%$ confidence interval. We observe that for $\lambda=1$ and for $n=4$ the probability of collision is already small (around 0.02), This probability can be extremely small for $n=10$ : around 0.0005 . In Figure 6 we also observe a very good matching between the analytical and simulation results.

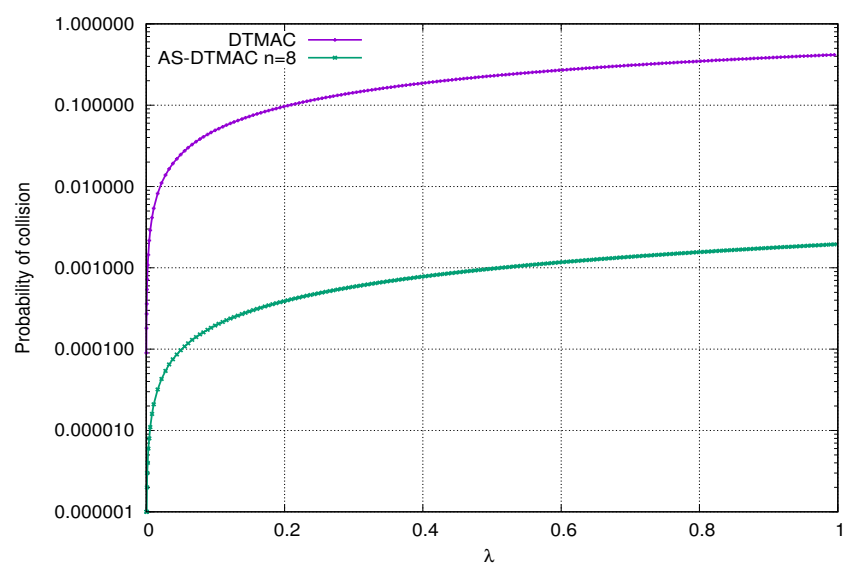

Fig. 7. Collision probability versus channel load.

In Figure 7, we compare the collision probability versus the channel load for DTMAC and for DTMAC enhanced by an active signaling scheme with $n=8$ mini-slots. We observe that the gain in the probability of collision is in the order of $10^{3}$. This is a huge gain which clearly shows the interest of the signaling scheme.

\section{B. Burst of traffic}

In this case we have a burst of $k$ arrivals of urgent (and sporadic) packets on a given slot and we assume that there are no other competitors. Such an effect can be obtained if we use a dedicated first bit in the transmission key. We can use a first bit set to 1 in the transmission key for the $k$ urgent packets whereas the first bit is set to 0 for the default packets. Doing so, after the first signaling interval, their will be competition between $k$ urgent packets. In this part we focus on the transmission of these $k$ urgent packets We use the simple persistent protocol in which each of the $k$ pending packets successively uses the coming slot until the packets are successfully transmitted. According to the computations above, the probability of collision when there are $k$ packets competing for a slot is:

$$
\epsilon_{k}=1-A_{k}(0)-A_{k}^{\prime}(0)
$$

We call $T_{k}$ the mean number of slots until the $k$ packets are successfully transmitted. We have the following recursion

$$
T_{k}=1+\left(1-\epsilon_{k}\right) T_{k-1}+\epsilon_{k} T_{k}
$$

Thus we have the following equation:

$$
T_{k}=T_{k-1}+\frac{1}{\left(1-\epsilon_{k}\right)}
$$

Similarly for $2 \leq j \leq k$ we obtain:

$$
T_{j}=T_{j-1}+\frac{1}{\left(1-\epsilon_{j}\right)}
$$

and of course $T_{1}=1$. The resolution leads to

$$
T_{k}=1+\sum_{j=2}^{k} \frac{1}{\left(1-\epsilon_{j}\right)}=\sum_{j=1}^{k} \frac{1}{\left(1-\epsilon_{j}\right)} .
$$

Note that since $\epsilon_{1}=0, \frac{1}{\left(1-\epsilon_{1}\right)}=1$.

The exact distribution of $T$, the necessary number of slots until all the $k$ transmissions are successful is given below:

$$
\begin{gathered}
\operatorname{Prob}(T=k)=\prod_{j=1}^{k}\left(1-\epsilon_{j}\right) \\
\operatorname{Prob}(T=k+m)=\prod_{j=1}^{k}\left(1-\epsilon_{j}\right) \sum_{m_{1}+\ldots+m_{k}=m} \epsilon_{1}^{m_{1}} \ldots \epsilon_{k}^{m_{k}}
\end{gathered}
$$

The proofs of these formulas are simple. For the first formula the probability is exactly the probability that during the $k$ successive transmissions there is no collision. Since at each transmission the number of contending nodes decreases by 1 , the result is straightforward. Note that $\epsilon_{1}=0$ is left for the symmetry of the formula. 
For the second formula the probability is exactly the probability that, to obtain $k$ successful transmissions, we encounter exactly $m$ collisions. We have to distribute these $m$ collisions into $m_{1}, \ldots, m_{k}$ collisions such as $m_{1}+\ldots+m_{k}=m$ and some transmissions $j$ can be without collision in that case $m_{j}=0$. Note that $\epsilon_{1}=0$ and that necessarily $m_{1}=0$ the terms $\left(1-\epsilon_{1}\right)$ and $\epsilon_{1}^{m_{1}}=1$ are left for the symmetry of the formula.

Figure 8 presents the cumulative distribution function of the number of slots required for a burst of 10 urgent packets for $n=6$ and $n=10$. We observe that AS-TDMAC is very quick to successfully send all the packets even with $n=6$.

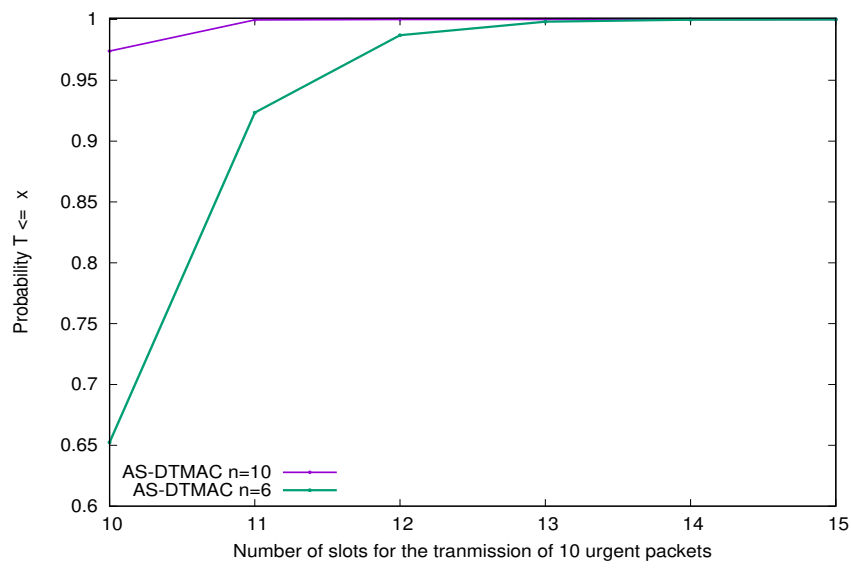

Fig. 8. Cumulative distribution function of the number of slots required for the 10 urgent packets.

\section{Analysis of the convergence speed to steady-state}

For DTMAC and DTMAC with active signaling, the access is organized in frames of slots. For the first frame we assume that the vehicles select a slot in the first frame at random. To simplify the analysis, we assume that the transmission attempts on the slots follow a Poisson process. Some competitors acquire a slot in this first frame. The competitors in collision during the first frame perform another attempt in the second frame in the free remaining slots.

\section{1) DTMAC with active signaling:}

This analysis is for the first transmission attempt when the vehicles compete to obtain a slot. We observe that the probability of collision is very small, which means that nearly all the slots where there is at least one transmission is a success. The mean number of successful transmissions on a given slot is $S_{r}$ :

$$
S_{r}(\lambda)=\sum_{k=1}^{\infty} \frac{\lambda^{k}}{k !} \exp (-\lambda) A_{n}^{k^{\prime}}(0) \simeq 1-\exp (-\lambda)
$$

We intialize $S_{r}=S_{r}(\lambda)$ which is the success rate during the first frame. We assume that the competitors that have not been able to find a slot during the first frame still form a Poisson process of rate

$$
\lambda-S_{r}
$$

but are competing on the slots not already acquired by the selection process. We compute the intensity of this process (step 1); it is:

$$
\lambda_{1}=\frac{\lambda-S_{r}}{1-S_{r}}
$$

and the success rate is:

$$
S_{r}\left(\lambda_{1}\right)
$$

and the additional packet success rate is:

$$
\begin{gathered}
S_{r}^{\prime}=\frac{S_{r}\left(\lambda_{1}\right)}{\lambda_{1}} *\left(\lambda-S_{r}\right) \\
S_{r}^{\prime}=S_{r}\left(\lambda_{1}\right) *\left(1-S_{r}(\lambda)\right) .
\end{gathered}
$$

We can continue the algorithm by updating $S_{r}$ :

$$
S_{r}=S_{r}+S_{r}^{\prime}
$$

which is the total success rate during the second frame. We can return to step 1 to compute the total success rate in the coming frame. The process continues iteratively.

\section{2) DTMAC alone:}

The analysis is the same except that the success rate with DTMAC appears when a slot is only requested by a single competitor, thus the success rate is:

$$
S_{r}(\lambda)=\lambda \exp (-\lambda)
$$

We initialize $S_{r}=S_{r}(\lambda)$ which is the success rate in the first frame. We compute the intensity of the competitors requesting a slot ${ }^{1}$ in the next frame: frame 2 (step 1), it is still:

$$
\lambda_{1}=\frac{\lambda-S_{r}}{1-S_{r}}
$$

and the success rate is:

$$
S_{r}\left(\lambda_{1}\right)
$$

and the additional packet success rate is:

$$
\begin{gathered}
S_{r}^{\prime}=\frac{S_{r}\left(\lambda_{1}\right)}{\lambda_{1}} *\left(\lambda-S_{r}\right) \\
S_{r}^{\prime}=S_{r}\left(\lambda_{1}\right) *\left(1-S_{r}(\lambda)\right) .
\end{gathered}
$$

We can continue the algorithm by updating $S_{r}$ :

$$
S_{r}=S_{r}+S_{r}^{\prime}
$$

We can return to step 1 to compute the total success rate in the coming frame. The process continues iteratively.

We can see in Figure 9 below the percentage of slots acquired by DTMAC and DTMAC with AS with respect to the index of the frame; the error bars in black are for a $95 \%$ confidence interval. We have assumed a highly loaded channel $(\lambda=0.96)$. DTMAC with AS requires 5 frames to successfully reserve the input load. DTMAC alone requires twice as many frames i.e. 10 frames. We note a good matching between the simulation and analytical results.

\footnotetext{
${ }^{1}$ not already acquired during the previous frames.
} 


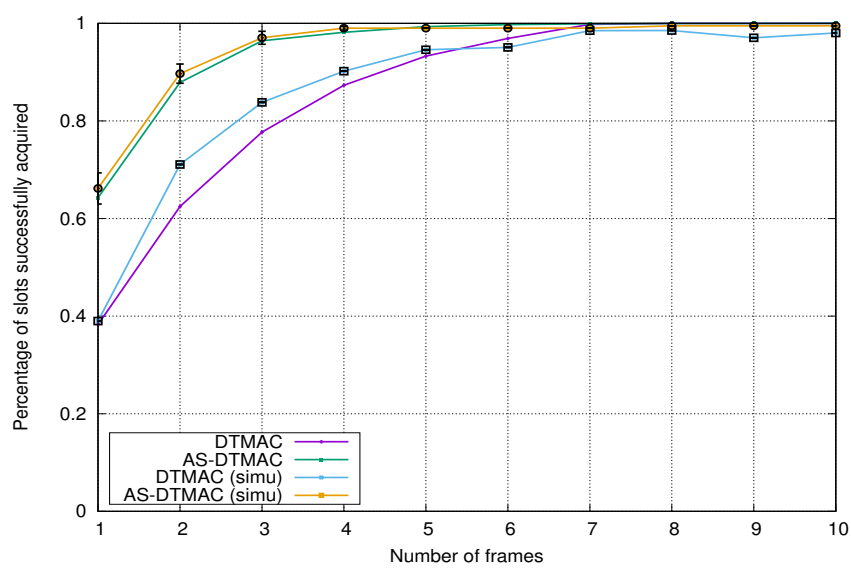

Fig. 9. Convergence towards the steady state, $\lambda=0.96$.

\section{CONCLUSION}

In this paper we propose an analytical model to analyze the AS-DTMAC protocol and to compare it with DTMAC. This model, based on the use of generating functions, can be very simply exploited to obtain very good performances. We study the collision rate of AS-DTMAC when the vehicles randomly select their slot in the time frame (the normal condition of the protocol). In this situation we also investigate the number of time frames needed to obtain a collision free slot for all the vehicles in the network. The transmission conditions of urgent packets which are sent persistently until successful are also studied with an analytical model. The distribution of the duration of such a process is computed.

The simulations confirm the results of the analytical model and the very good performances of AS-DTMAC in terms of collisions and convergence to a steady state. The transmission of urgent packets is also very efficient. The exact definition of the signaling bursts will be the subject of future work with the computation of miss detection in the selection process. This work is currently in progress.

\section{REFERENCES}

[1] Fei Hu, "Security and Privacy in Internet of Things (IoTs): Models, Algorithms, and Implementations", ISBN 978-1-4987-2318-3.

[2] M. Hadded, P. Muhlethaler, A. Laouiti, R. Zagrouba, and L. A. Saidane, "TDMA-based MAC protocols for vehicular ad hoc networks a survey, qualitative analysis and open research issues", IEEE Communications Surveys Tutorials, vol. 17, no. 4, pp. 2461-2492, Jun. 2015.

[3] M. Hadded, P. Muhlethaler, A. Laouiti and L. A. Saidane, "An Infrastructure-Free Slot Assignment Algorithm for Reliable Broadcast of Periodic Messages in Vehicular Ad Hoc Networks", 2016 IEEE 84th Vehicular Technology Conference (VTC-Fall), Montreal, QC, Canada, pp. 1-7, Sept. 2016.

[4] F. Boukhalfa, M. Hadded, P. Muhlethaler and O. Shagdar, "An active signaling mechanism to reduce access collisions in a distributed TDMA based MAC protocol for vehicular networks", Advanced Information Networking and Applications (AINA-2019), Matsue, Japan, pp. 286-300, Mar. 2019.

[5] F. Ye, R. Yim, J. Zhang, and S. Roy, "Congestion control to achieve optimal broadcast efficiency in VANETs", in IEEE International Conference on Communications (ICC), Cape Town, South Africa, May 2010, pp. 1-5.
[6] 802.11p-2010, IEEE standard for information technology - Telecommunications and information exchange between systems - local and metropolitan area networks - specific requirements part 11: Wireless LAN medium access control (MAC) and physical layer (PHY) and physical layer (PHY) specifications amendment 6 : Wireless access in vehicular environments Std., 2010.

[7] R. Uzcategui and G. Acosta-Marum, "Wave: A tutorial”, IEEE Communications Magazine, vol. 47, no. 5, pp. 126-133, May 2009.

[8] F. Borgonovo, A. Capone, M. Cesana, and L. Fratta, "ADhoc MAC: new mac architecture for ad hoc networks providing efficient and reliable point-to-point and broadcast services", Wireless Networks, vol. 10, no. 4, pp. 359-366, 2004.

[9] F. Borgonovo, A. Capone, M. Cesana, and L. Fratta, "Rr-Aloha, a reliable r-Aloha broadcast channel for ad-hoc inter-vehicle communication networks", in IEEE IFIP Annual Mediterranean Ad Hoc Networking Workshop (Med-Hoc-Net), Baia Chia, Italy, 2002.

[10] W. Ke, Y. Weidong, L. Pan, and Z. Hongsong, "A decentralized adaptive TDMA scheduling strategy for VANET", in IEEE Wireless Communications and Networking Conference Workshops (WCNCW), Shanghai, China, Apr. 2013, pp. 216-221.

[11] Y. Weidong, L. Pan, L. Yan, and Z. Hongsong, "Adaptive TDMA slot assignment protocol for vehicular ad-hoc networks", Journal of China Universities of Posts and Telecommunications, vol. 20, no. 1, pp. 1118, Feb. 2013.

[12] F. Karnadi, Z. Mo, and K. chan Lan, "Rapid generation of realistic mobility models for VANET", in IEEE WCNC, Hong Kong, China, Mar. 2007, pp. 2506-2511.

[13] SUMO Simulator: http://sumo.sourceforge.net. 